\title{
A study of particles penetration in sieving process on a linear vibration screen
}

\author{
Zhanfu Li ${ }^{1} \cdot$ Xin Tong ${ }^{1}$
}

Received: 16 November 2014/Revised: 15 December 2014/Accepted: 16 March 2015/Published online: 9 December 2015

(C) The Author(s) 2015. This article is published with open access at Springerlink.com

\begin{abstract}
This paper presented an investigation of particle collision and penetration using the discrete element method to understand the motion of particles and improve theoretical treatment in the sieving process. The process progressively was divided into looseness, stratification, collision, and penetration. Particle penetration has a direct effect on the screening performance. The penetration probability was defined, and the mathematical relationships between particle penetration and vibration parameters were established using the least squares method. To obtain the ideal penetration probability for materials the amplitude and frequency should preferably be near $3.0 \mathrm{~mm}$ and $25 \mathrm{~Hz}$, respectively. The vibration direction angle has only a slight effect on penetration. The stage of the screening process from 0.1 to $0.7 \mathrm{~s}$ is the primary region for collision and penetration. This paper focused on the sieving process to more fully understand how particle collision and penetration influence the screening efficiency.
\end{abstract}

Keywords Penetration probability $\cdot$ Collision $\cdot$ Discrete element method $\cdot$ Sieving process

\section{Introduction}

The understanding of screening process is incomplete regarding the intricacies of particle-particle and particlemachine interactions. But the influence of these interactions on the screening process effects on both the efficiency and the value of the product (Soldinger 2000; Zhao et al. 2000; Jiao et al. 2006). We divide the screening process into four parts as follows: (1) looseness: the void space that occurs between particles which are rebounded by the sieve plate. A sufficient void space contributes to particle stratification (Zhang and Tong 2012). (2) Stratification: the ability of the fine particles to penetrate the coarse granules, when all particles are scattered. In other words, stratification relates to the ability of the fine particles to approach the screening surface (Xiao and Tong 2012). (3) Collision:

Xin Tong

ccq@hqu.edu.cn

1 School of Mechanical Engineering and Automation, Huaqiao University, Xiamen 361021, China once close to the screening surface, some particles impact the sieve wires and some pass through the sieve mesh. These particles that are near the mesh are referred to as the collision particles (Wei et al. 1999). (4) Penetration: the penetration rate is the fraction of the collision particles which penetrate the mesh. It has a direct effect on screening performance.

Some previous research has developed our current understanding and theory of single grain screen-penetrating probability. The probability model and equations for a spherical particle penetrating a hole were developed by Subasinghe et al. (1989). Wen and Liu (1982) put forward various characteristic motions that a single particle on the sieve surface may undertake: relative static, relative sliding and projectile motion. Applying chaotic motion theory to analyze the movement of the single particle, Liu and $\mathrm{Lu}$ (2009) concluded that the motion is nonlinear. Beyond the single particle, theory of particle cluster screen-penetrating probability has also been established. In 1972, the collision speed transfer formula of particle clusters in the sieving process was established by Bruder. Using the method of probability statistics, the mathematical model of the 
penetration probability along the screen was put forth by Chen and Yan (1982). In recent years, discrete element method (DEM) allows for modeling at the particle level and accounts for interaction between particles and boundary objects. Cleary (2004) and Cleary and Sawley (2002) presented a three dimensional model of spherical particle separation on an inclined flat screen based on DEM. Tong explored the relationship between vibration parameters and screen efficiency, as well as performing simulations of stratification and penetration over various structural and vibration parameters based on the DEM (Chen and Tong 2009, 2010; Wang and Tong 2011). The implementation of DEM for screening process gives an insight to study the particulate systems. DEM simulations have been reported positive results in mineral screening and crop cleaning (Zhu et al. 2008; Weerasekara et al. 2013; Cleary and Dsinnott 2015; Cleary 2015; Xue et al. 2015).

Taking into account particle-particle and particle-machine interactions, we study herein a series of screening simulations which provides a theoretical foundation for apparatus design based on optimized screening parameters, and provides a reasonable explanation for the screening process.

\section{Simulation}

Successful application of the DEM, which is a numerical method suitable for tracking particle characteristics including speed and displacement, to mineral processing and material separation represents a good way to optimize design of vibration screens. However, few studies about DEM simulations of the screening process have been reported.

\subsection{The contact model used in the simulation}

The model is based on the work of Mindlin (Mindlin and Deresiewicz 1953). The elastic contact occurs in two spherical granules whose radii are $R_{1}$ and $R_{2}$ respectively. $R^{*}$ is the equivalent radius. The normal overlap, $\alpha$ is given by

$\alpha=R_{1}+R_{2}-\left|r_{1}-r_{2}\right|$

$\frac{1}{R^{*}}=\frac{1}{R_{1}}+\frac{1}{R_{2}}$

with the centers of the two particles position vectors are given by $r_{1}$ and $r_{2}$. The normal force $F_{n}$, is given by

$F_{n}=\frac{4}{3} E^{*} \sqrt{\alpha^{\frac{3}{2}} R^{*}}$

$E^{*}=\frac{1-\gamma_{1}^{2}}{E_{1}}+\frac{1-\gamma_{2}^{2}}{E_{2}}$

where $E^{*}$ is the equivalent Young's modulus. $E_{1}, \gamma_{1}$ and $E_{2}$, $\gamma_{2}$ are the Young's modulus and Poisson's ratio for particles 1 and 2, respectively. Additionally there is a damping force, $F_{n}^{d}$, given by

$F_{n}^{d}=-2 \sqrt{\frac{5}{6}} \beta V_{n}^{\mathrm{rel}} \sqrt{S_{n} m^{*}}$

$\beta=\frac{\ln e}{\sqrt{\ln ^{2} e+\pi^{2}}}$

$S_{n}=2 E^{*} \sqrt{\alpha R^{*}}$

$V_{n}^{\mathrm{rel}}=\left(v_{1}-v_{2}\right) n$

where $m^{*}$ is the reduced mass, $V_{n}^{\text {rel }}$ is the normal component of the relative velocity. The two particles' speeds are $v_{1}$ and $v_{2}$. The tangential force, $F_{t}$ depends on the tangential overlap $\delta$, and the tangential stiffness, $S_{t}$.

$F_{t}=-\delta S_{t}$

$S_{t}=8\left(\frac{2-\gamma_{1}^{2}}{G_{1}}+\frac{2-\gamma_{2}^{2}}{G_{2}}\right) \sqrt{\alpha R^{*}}$

additionally, tangential damping is given by:

$F_{t}=-2 \sqrt{\frac{5}{6}} \beta V_{t}^{\mathrm{rel}} \sqrt{m^{*} S_{t}}$

where $V_{t}^{\text {rel }}$ is the relative tangential velocity. $G_{1}$ and $G_{2}$ are the shear modulus of particles 1 and 2, respectively.

\subsection{Simulation model and conditions}

The 3D vibration screening model was set up to simulate the linear vibration based on DEM. In simulations, the particles were produced by particle factory (Fig. 1). The mode of screen surface was set by software EDEM. The discharge port saved the particles which did not pass the aperture. This paper focused on particles collision and penetration over the screen surface in the process of screening (Fig. 2). The three-dimensional (3D) model was used to simulate the movement of all particles in the

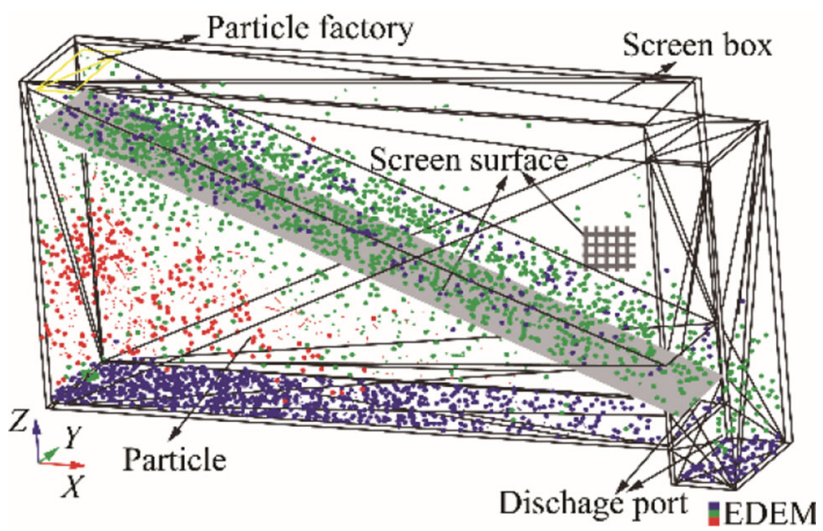

Fig. 1 DEM-3D model 
screening process (Fig. 3). Because interactions happen between the particle and the screen surface, the springdashpot-slider model was used to simulate particles collision (Soliding 1999). The DEM model has been shown to provide good agreement between numerical and experimental results (Dong et al. 2009). The screen body is $162 \mathrm{~mm}$ long, $32 \mathrm{~mm}$ wide and $80 \mathrm{~mm}$ high. Meanwhile, considering the size distribution of sands used in the industry, this paper employed a bimodal normal distribution of particles which are provided by the particle supply (Fig. 1) with a standard deviation of 0.545 . The particles are spherical sand with mean diameters of 0.5 and $1.0 \mathrm{~mm}$. As shown in Fig. 2, $\beta$ is the vibration direction angle; $\alpha$ is the inclination of the screen surface; $A$ is the amplitude. In Fig. 1 the position of the particles are presented. In this model, the particles fall down to sieve plate constantly by gravity. The process of sieving simulation was presented on Fig. 3. The vibration parameters are mainly frequency, amplitude and vibration direction angle. Simulation conditions are summarized in Tables 1 and 2. Three type experiments have been accomplished. The particle generate rate is 42,000 particle/s, the screen declination is $21^{\circ}$, screen vibration is sinusoidal translation, the total number is 30,000. Each groups of experiments only changed one parameter value and the conditions are listed in Table 3. Such as group one, only amplitude is varying.

\section{Definition of penetration probability and discussion}

During the screening process simulation, the particles took $0.3 \mathrm{~s}$ to move to the end of the screen surface and took over $1.3 \mathrm{~s}$ to complete screening. In this study, the penetration probability is defined as the ratio of the mass of those particles which penetrate the sieve plate to the total mass of all collision particles over the time period from 0.4 to $0.6 \mathrm{~s}$. From the Fig. 2, the blue particles are called the

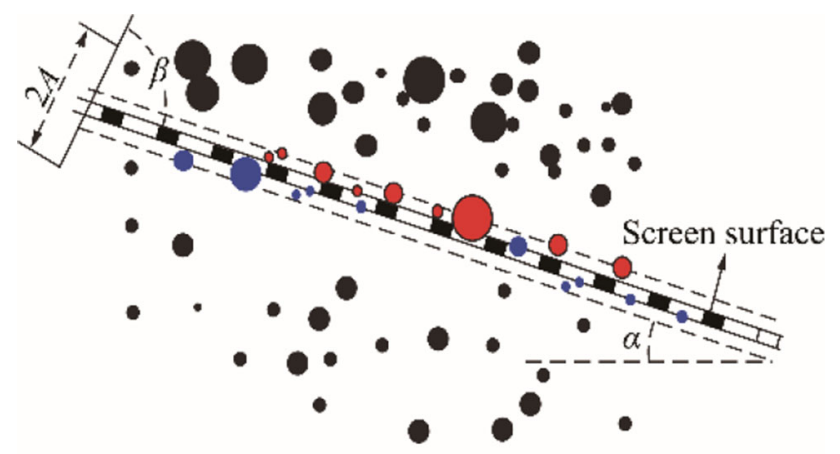

Tाग

Fig. 2 Schematic side-view of the screen surface

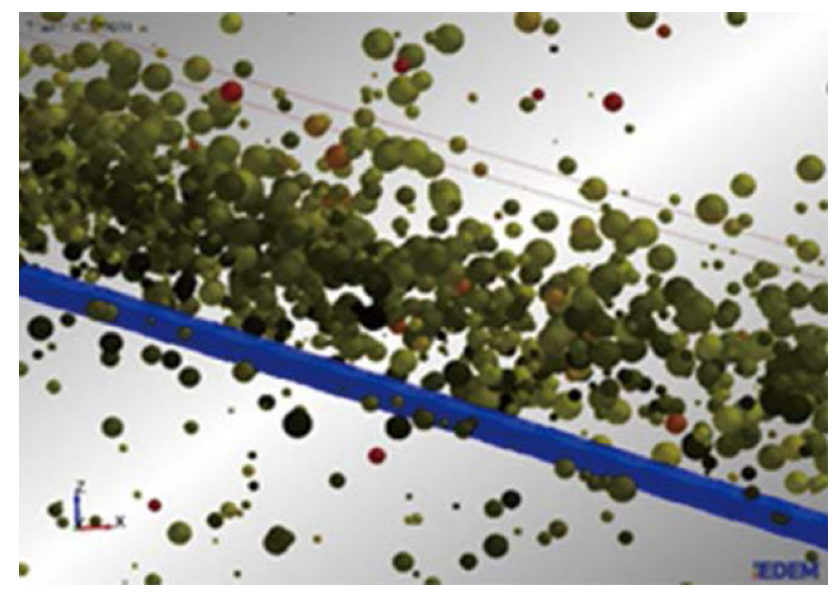

Fig. 3 Process of sieving simulation

penetration particles and the particles between the two dotted lines are the collision particles. The distance between the dotted line and the screening surface is the diameter of the largest particle. Because the penetration and collision particles have a direct effect on efficiency, the penetration and collision particles vary in different vibration parameters. The penetration probability is given by

$T=\frac{M_{\mathrm{p}}}{M_{\mathrm{c}}} \times 100 \%$

where $M_{\mathrm{p}}$ is the penetration particles' mass, $M_{\mathrm{c}}$ is the mass of the collision particles.

This study forces on the effects of vibration parameters, including amplitude, frequency and vibration direction angle, on the penetration probability.

\subsection{Frequency effects on penetration probability}

Figure 4a shows the number of collision particles over time at different frequencies. At high frequency $(f>40 \mathrm{~Hz})$ the changes in the particle number is not as obvious as at the lower frequencies and few particles have an opportunity to penetrate the apertures at high frequency (Fig. 4b). The time from the beginning of charging to $0.7 \mathrm{~s}$ is the main period of collision and penetration. The relationship between frequency and penetration probability is presented in Fig. 4c. The penetration probability decreases as the size of separated particles increases and the maximum probability occurs at a frequency between 20 and $25 \mathrm{~Hz}$.

From Fig. 4b, c, it can be seen that the mass of penetration particles increases, but the penetration probability decreases when the frequency is greater than about $25 \mathrm{~Hz}$. This illustrates that the increment speed of the collision particles is greater than the penetration particles. Meanwhile, because the increment speed of the collision particles is less than that of the penetration particles when the frequency is less than $20 \mathrm{~Hz}$, the penetration probability 
Table 1 Material properties setting in EDEM

\begin{tabular}{llll}
\hline Material properties & Poisson's ratio & Shear modulus & Density $\left(\mathrm{kg} / \mathrm{m}^{3}\right)$ \\
\hline Particles & 0.3 & $23 \mathrm{MPa}$ & 2678 \\
Screen & 0.29 & $79.92 \mathrm{GPa}$ & 7861 \\
\hline
\end{tabular}

Table 2 Collision properties setting in EDEM

\begin{tabular}{llll}
\hline Collision properties & Coefficient of restitutions & Coefficient of static friction & Coefficient of rolling friction \\
\hline Particle-particle & 0.1 & 0.545 & 0.01 \\
Particle-screen & 0.2 & 0.5 & 0.01 \\
\hline
\end{tabular}

Table 3 Summary of conditions

\begin{tabular}{llll}
\hline Text group & Amplitude $(\mathrm{mm})$ & Frequency $(\mathrm{Hz})$ & Vibration angle \\
\hline Group one & $0.5,1.5,2,2.55,3.5$ & 20 & 100 \\
Group two & 2.55 & $10,20,25,30,40,50$ & 100 \\
Group three & 2.55 & 20 & $10,20,30,40,50,60,70,80$ \\
\hline
\end{tabular}

curves have an upward trend. When $f>40 \mathrm{~Hz}$, the particles completely fill the screening box. At last, the data in Fig. $4 \mathrm{c}$ were fit, with low relative error, to the function:

$y=(a+b x) e^{-c x}$

where $y$ is penetration probability, $x$ is frequency, $a, b$, and $c$ are fitting parameters, depending on particle separation diameter (Fig. 4c).

\subsection{Amplitude effects on penetration probability}

Figure 5a shows the number of collision particles over time for different amplitudes. At low amplitudes, because most particles are close to the sieve plate, the collision numbers obviously increase. At high amplitudes, the particles can gain more energy to be rebounded. The contacts between the sieve plate and particles decrease. The number of penetration particles over time is shown in Fig. 5b. The time from the beginning of charging to $0.7 \mathrm{~s}$ is the main region of collision and penetration, after which the slope of the change in number of penetration particles decreases. As the amplitude increases, the number of penetration particles decreases. We chose the statistical window to determine the penetration probability to be from the time at which the most particles are scattered evenly to the end time of feeding, that is, from 0.4 to $0.6 \mathrm{~s}$.

From Fig. 5c we see that the amplitude of $2.55 \mathrm{~mm}$ gives the highest value. Because of the speed of change about the numerator and denominator of Eq. (12), the curve presents that as shown in Fig. 5c. Data obtained with amplitudes less than $3.55 \mathrm{~mm}$ were fit to the following function, with low relative error:

$y=e^{c x^{2}+b x+a}$

where $x$ is the amplitude, $y$ is the penetration probability, and $a, b$, and $c$ are fitting constants dependent on the particle diameter (Fig. 5c).

\subsection{Vibration direction angle effects on penetration probability}

Figure $6 a$ demonstrates that the mass of the collision particles does not change much for different vibration direction angles. Unlike amplitude and frequency, the particles do not gain enough energy to jump and be thrown over the screen surface, and the vibration direction angle has only a slight effect on the mass of the collision. Figure 6 a shows at the angle of 20 and 31, the particles' velocity component, which is perpendicular to screen surface is small, so they return to the sieve plate quickly. The change of the penetration particles' number over time is presented on Fig. 6b. In addition the difference is little among these different vibration angles. On the whole, the effect of vibration angle differs from that of the amplitude and frequency. The vibration angle has a more subtle effect than others (Fig. 6c). 

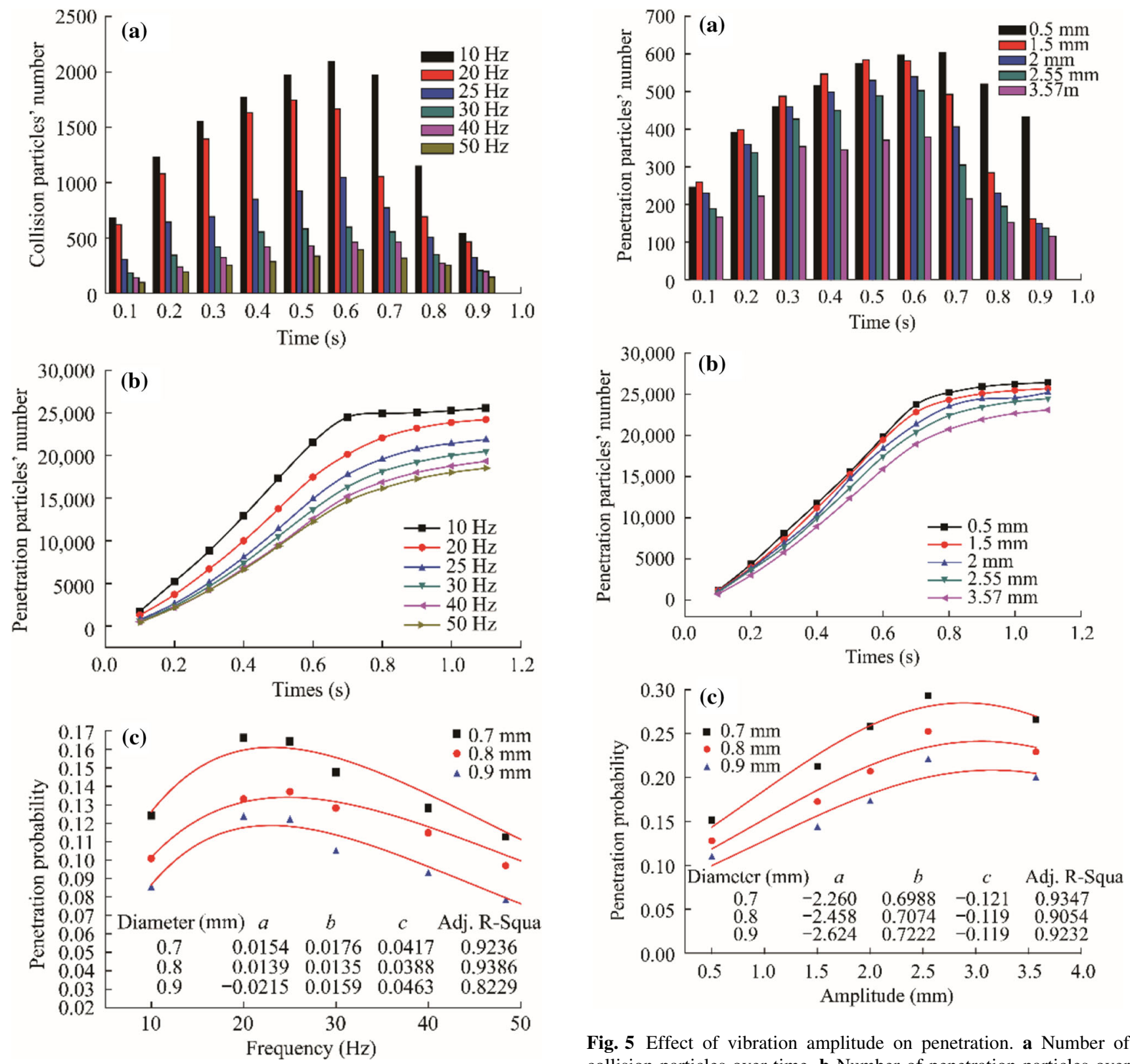

Fig. 4 Effect of vibration frequency on penetration. a Number of collision particles over time, $\mathbf{b}$ Number of penetration particles over time, $\mathbf{c}$ Penetration probability versus frequency

The relationships between penetration probability and vibration parameters were presented. The main conclusions show that to obtain the ideal penetration probability for materials the amplitude and frequency should be chosen to be about $3.0 \mathrm{~mm}$ and $25 \mathrm{~Hz}$, respectively. The vibration direction angle has a slight effect on the penetration probability. This paper focuses on the particles penetration under various single parameter experiments using the DEM and provides insight about screening process and a theoretical foundation for designing the screen equipment.

Fig. 5 Effect of vibration amplitude on penetration. a Number of collision particles over time, b Number of penetration particles over time, $\mathbf{c}$ Penetration probability versus amplitude

\section{Conclusions}

(1) Simulations were used to establish changes in the number of penetration and collision particles over time. Because of the complex sieving process which is hard to gain the information from the sieving experiments, we simulated these experiments using the method of DEM which has been shown to provide good results for similar problems. The effects of vibration conditions on the sieving processes have been studied. 

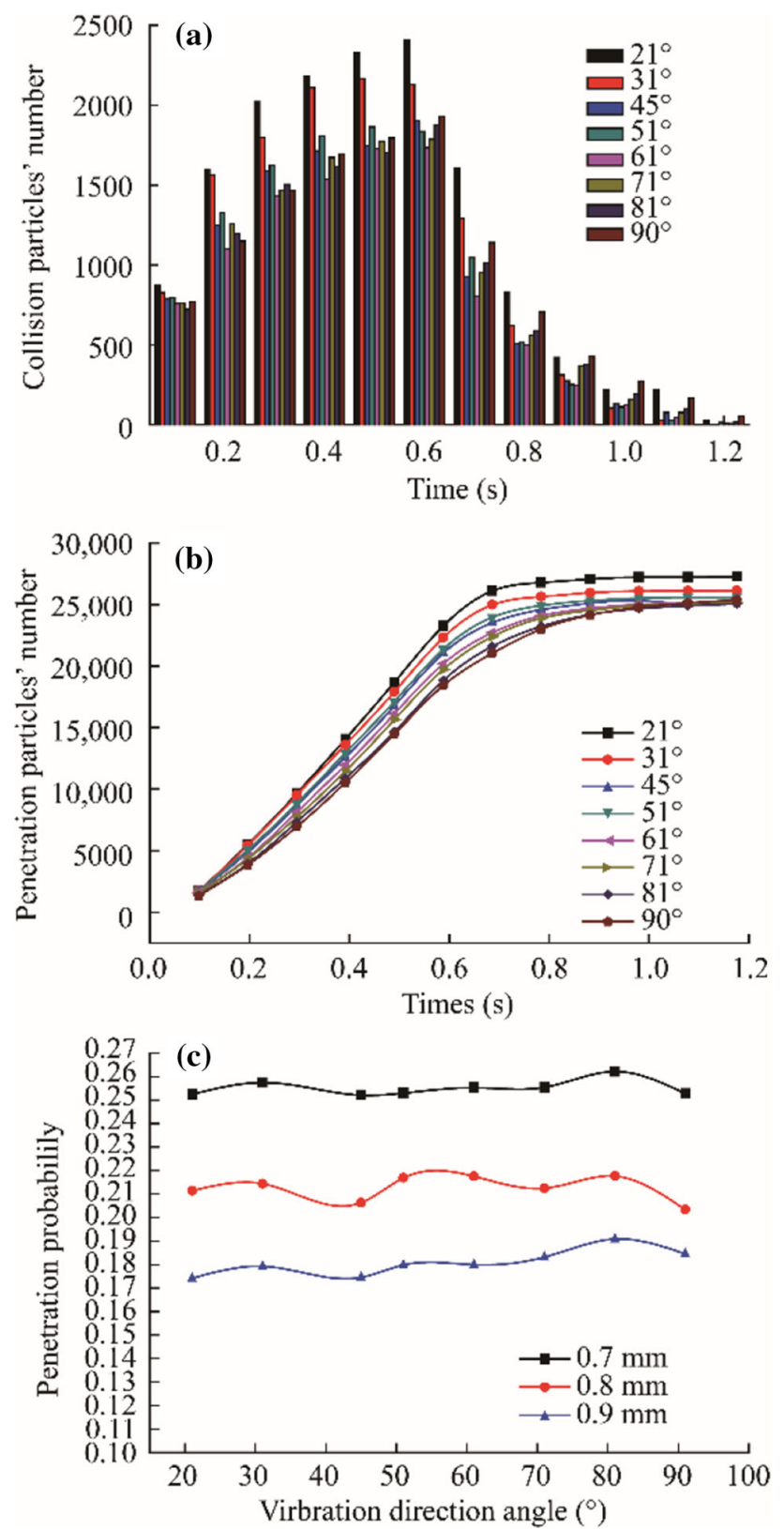

Fig. 6 Effect of vibration direction angle on penetration. a Number of collision particles over time, b Number of penetration over time, c Penetration probability versus vibration direction angle

(2) The results show that the stage of the screening process from 0.1 to $0.7 \mathrm{~s}$ is the main part for collision and penetration. The number of penetration particles mounted and leveled off. In a representative continuous screening process, the relationships between the penetration probability and the vibration parameters were modeled. To obtain the ideal penetration probability for materials the amplitude and frequency should be chosen to be about $3.0 \mathrm{~mm}$ and $25 \mathrm{~Hz}$, respectively. The vibration direction angle has a slight effect on the penetration probability.
(3) The sieving process was divided into four stages as follows: looseness, stratification, collision and penetration. Each part is important to screen performance. Further research will focus on the sieving process to provided significant insight into design and manufacture vibration screen.

Acknowledgments The authors gratefully acknowledged the support from the National Natural Science Foundation of China (51175190).

Open Access This article is distributed under the terms of the Creative Commons Attribution 4.0 International License (http://crea tivecommons.org/licenses/by/4.0/), which permits unrestricted use, distribution, and reproduction in any medium, provided you give appropriate credit to the original author(s) and the source, provide a link to the Creative Commons license, and indicate if changes were made.

\section{References}

Chen YH, Tong X (2009) Application of the DEM to screening process: a 3D simulation. Min Sci Technol 19(4):493-497

Chen YH, Tong X (2010) Modeling screening efficiency with vibrational parameters based on DEM 3D simulation. Min Sci Technol 20(4):615-620

Chen QR, Yan F (1982) Theory and practice of the probability classification screen for coal. J China Univ Min Technol 2:25-33

Cleary PW (2004) Large scale industrial dem modeling. Eng Comput 21:169-204

Cleary PW (2015) Prediction of coupled particle and fluid flows using DEM and SPH. Miner Eng 73:85-99

Cleary PW, Dsinnott M (2015) Simulation of particle flows and breakage in crushers using DEM: part 1-compression crushers. Miner Eng 74:178-197

Cleary PW, Sawley M (2002) DEM modelling of industrial granular flows: 3D case studies and the effect of particle shape on hopper discharge. Appl Math Model 26(2):89-111

Dong KJ, Yu AB, Brake I (2009) DEM simulation of particle flow on a multi-deck banana screen. Miner Eng 22(11):910-920

Jiao HG, Ma J, Zhao YM, Chen LJ (2006) Study on the numerical simulation of batch sieving process. J Coal Sci Eng 12(2):80-83

Liu CS, Lu JX (2009) Study on nonlinear characteristic of particle motion during the process of coal screening. J China Coal Soc 34(4):556-559

Mindlin RD, Deresiewicz H (1953) Elastic sphere in contact under varying oblique forces. J Appl Mech 20(3):327-344

Soldinger M (2000) Influence of panicle size and bed thickness oil the screening process. Miner Eng 13(3):297-312

Soliding M (1999) Interrelation of stratification and passage in the screening process. Miner Eng 12(5):497-516

Subasinghe GKNS, Schaap W, Kelly EG (1989) Modeling the screening process: a probabilistic approach. Powder Technol 59(1):37-44

Wang GF, Tong X (2011) Screening efficiency and screen length of a linear vibrating screen using DEM 3D simulation. Ming Sci Technol 21(3):451-455

Weerasekara NS, Powell MS, Cleary PW, Tavares LM, Evertsson M, Morrison RD, Quist J, Carvalho RM (2013) The contribution of DEM to the science of comminution. Powder Technol 248:3-24

Wei LB, Zhao YM, Chen QR (1999) Study on the parameters by imitating the process of probability screen for coal sizing. J China Coal Soc 24(1):103-107 
Wen BC, Liu FQ (1982) Vibration machine theory and application. China Machine Press, Beijing

Xiao JZ, Tong X (2012) Particles stratification and penetration of a linear vibrating screen by the discrete element method. Int J Min Sci Technol 22(3):357-362

Xue S, Yuan L, Wang JF, Wang Y, Xie J (2015) A coupled DEM and LBM model for simulation of outbursts of coal and gas. Int $\mathrm{J}$ Coal Sci Technol 2(1):22-29
Zhang EL, Tong X (2012) Study on loose mechanism of vibrating screen based on DEM. Min Process Equip 40(12):67-70

Zhao LL, Liu CS, Yan JX (2010) Min Sci Technol 20(2):276-280

Zhu HP, Zhou ZY, Yang RY, Yu AB (2008) Discrete particle simulation of particulate systems: a review of major applications and findings. Chem Eng Sci 63(23):5728-5770 